\title{
Contact Resistance Evolution of Au-Au Micro- Contacts with encapsulated Ag colloids
}

\author{
Benjamin Toler, Christopher Stilson and Ronald Coutu, Jr. \\ Department of Electrical and Computer Engineering \\ Air Force Institute of Technology \\ Wright Patterson AFB, Ohio, 45433
}

\begin{abstract}
This paper reports the contact resistance evolution results of thin film, sputtered gold with encapsulated Ag colloids, micro-contacts dynamically tested up to $3 \mathrm{kHz}$. The upper contact support structure consists of a sputtered gold surface micromachined, fixed-fixed beam designed with sufficient restoring force to overcome adhesion. The hemisphere-upper and planar-lower contacts are mated with a calibrated, external load resulting in approximately $100 \mu \mathrm{N}$ 's of contact force. Contact resistance is measured, in-situ, using a cross-bar configuration and the entire apparatus is isolated from external vibration and housed in an enclosure to minimize contamination due to ambient environment. Additionally, contact cycling and data collection are automated using a computer and LabVIEW. The results showed that $\mathrm{Au}-\mathrm{Au}$ micro-contact had a final resistance of $14 \Omega$ s after 10 million cycles and $81 \Omega$ s after 8 million cycles with Au-Ag micro-contacts.
\end{abstract}

Index Terms-Micro-Contacts; Reliability; Performance; AuAu micro-contacts; Contact Resistance;

\section{INTRODUCTION}

Reliable microelectromechanical systems (MEMS) switches are critical for developing high performance radio frequency circuits like phase shifters. Engineers have attempted to improve reliability and lifecycle performance using novel contact metals, unique mechanical designs and packaging. Various test fixtures including: MEMS devices, atomic force microscopes (AFM) and nanoindentors have been used to collect resistance and contact force data. MEMS devices allow for lifecycle testing but not direct measurement of contact force which must be inferred through modeling. AFM and nanoindentor test fixtures allow direct contact force measurements but are severely limited by low resonance sensors.

This paper describes the research methodology used to explore and characterize the physics and evolution of microcontacts over their lifetime of performance. Understanding

Disclaimer: The views expressed in this article are those of the authors and do not reflect the official policy or position of the United States Air Force, Department of Defense, or the U. S. Government. how the physics of the micro-contact interface evolves will enhance micro-contact development time and help predict lifetime performance. To accomplish this research, the following was required: to improve a test fixture to examine lifetime evolution characteristics of a micro-contact and to generate a description of the witnessed micro-contact evolution phenomena. In reference to improving a microcontact test fixture, the specific goal was to surpass the currently reported micro-contact testing rate of $800 \mathrm{~Hz}$ and achieve a rate more conducive to lifecycle testing [1]. After witnessing the performance evolution of micro-contacts, the specific goal was to find an accurate description for the changing micro-contact interface physical phenomena.

\section{TEST FIXTURE}

\section{A. Previous Methods}

Previous systems employ the use of an AFM to apply a known contact force. However, the AFM limits the rate at which the micro-contacts can be cycled for testing [2]. For this experiment, the use of a dedicated force sensor and piezo electric motor was expected to surpass the rate limitations of the AFM [1]. The ability to apply a known contact force in addition to known current enables greater accuracy for determining micro-contact resistance and is the reason that AFMs are employed for testing [2]. By using a piezo motor with a high-rate capable force sensor instead of an AFM, there was no expected decrease in micro-contact resistance accuracy. Instead, the rate of micro-contact testing was only limited to the capability of the piezo controller and data acquisition system, which was expected to be around $3 \mathrm{kHz}$.

To witness the evolution of micro-contact performance, micro-contact resistance was examined. Micro-contact resistance is directly impacted by interface changes and is a measurable quantity. In literature, assuming contaminant free surfaces, contact resistance is typically a function of four factors: the total contact area, material hardness, impact velocity, and contact force [3]. A change in any of the aforementioned factors can negatively impact performance. Other reasons for a decrease in performance are described as the development of surface contaminants, from oxide growth 
to the development of organic films [4]. Containment film development is described as the leading cause for a decrease in micro-contact performance [5]. However, these film developments change the performance of the micro-contact by changing the fundamental factors for micro-contact resistance. Previously, the development of thin films on micro-contacts was experimentally verified as having an impact on microcontact resistance [6]. In this research, a systematic approach was taken to examine and describe containment film development as it relates to the evolution of micro-contact performance.

\section{B. Test Stand}

The test stand for this research is made of several components to enable characterization of the performance evolution of a micro-contact over its lifetime. One requirement is the ability for visual confirmation of a force sensor in contact with a micro-contact test structure. Another necessity is an enclosed space for a nitrogen environment in which to test the micro-contact. Two Agilent 34410A multimeters are used to measure the current and voltage across the micro-contact. An Agilent U3606A current source is required to apply current to the micro-contact structure. A Femto Tools FT-S270 force sensor is used to determine the amount of force applied to the micro-contact test structure. In order to apply force to the micro-contact, a Thorlabs BPC301 piezo motor and controller is needed to move the sensor into and away from the micro-contact test structure. An Agilent 33250A waveform generator is programmed to supply the driving signal to the piezo actuator for high cycle rate testing. Micromanipulators are necessary for positioning the force sensor in alignment to the micro-contact test structure. In addition, the micro-contact support structure, which is fabricated on a silicon wafer, is placed on a carrier and fixed into place to allow for proper alignment and testing with the force sensor. To integrate these components into one system, a computer with LabVIEW and various computer interfaces were used.

Beginning with the micro-test structure fixture, a fabricated device on a wafer is fashioned to a carrier using a crystal bond. The micro-contact test support structure is then wire bonded to the pinouts of the carrier to make it easier to measure the current and voltage across the contact. This also reduces the probability of physically interacting and changing the surface of the micro-contact test structure by removing the necessity for probes. With the wafer wire bonded to the carrier, the carrier is then placed into the carrier socket which has pins for every wire bond. These pins are wire wrapped separately and the wires are guided outside of the enclosed test fixture. The carrier socket is fixed vertically into place by a metal bracket attached to a z-axis micro-manipulator. The manipulator is fixed to the bottom of the enclosed test fixture to prohibit movement in the $\mathrm{x}$ or $\mathrm{y}$ axis direction.

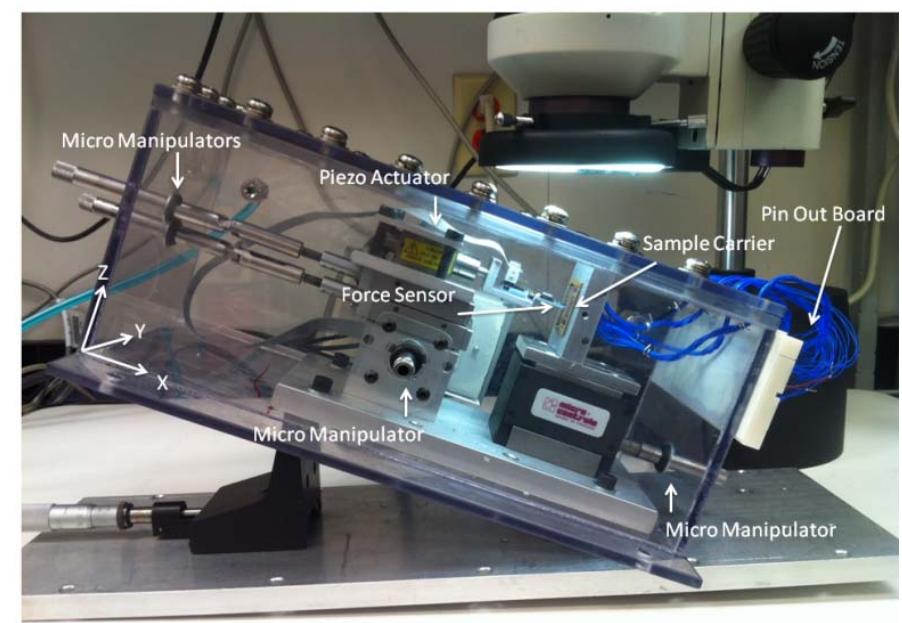

Fig. 1 Test Stand, Showing Major Componints, Which Is Encased In A Nitrogen Environment

Also fixed to the enclosed test fixture are two x-axis micromanipulators and one y-axis micro-manipulator opposite the $\mathrm{z}$-axis micro-manipulator. These $\mathrm{x}$-axis manipulators are fixed into place to prevent any movement in the $\mathrm{y}$ and $\mathrm{z}$ axis direction. The y-axis micro-manipulator allows for alignment in the y-axis direction. The piezo motor is attached to the two $\mathrm{x}$-axis and one $\mathrm{y}$-axis micro-manipulators. The piezo motor is fixed into place to allow movement solely in the $\mathrm{x}$-axis direction. The force sensor is attached to the piezo motor. As shown in Fig. 1, the force sensor, piezo motor, x-axis and yaxis micro-manipulators are stacked to allow the force sensor to be aligned with and actuate the micro-contact test structure in the carrier.

The enclosed test fixture provides the ability to create an inert (dry nitrogen) ambient environment. Testing in a nitrogen environment reduces the opportunity for oxides and other organic films to develop prematurely. The nitrogen environment is created to simulate a hermetic environment that a micro-contact would normally perform in. This test fixture allows for the knobs of all the micro-manipulators as well as the wires attached to the carrier socket to be accessible from outside the fixture as shown in Fig. 1.

\section{Testing Methods}

There are three types of contact testing which can be performed in order to characterize the evolution of performance for a micro-contact: virgin contact testing, hot switching, and cold switching. Virgin contact testing enables the determination of the appropriate contact force to be applied for reasonably low resistance. The applied contact force determined by the virgin contact test is then used for hot switching and cold switching tests. Hot switching increases the probability of electrical failure modes by cycling the micro-contact with constantly applied current. Cold switching, on the other hand, increases the probability of physical failure modes and only applies current during the time that the microcontact is actuated closed. 


\section{1) Virgin Contact Testing}

The virgin contact test applies a load to the micro-contact and determines the amount of force needed for the microsurfaces to be in contact. This applied force, required for a closed connection, is marked as the offset for applied contact force. All force to be applied from the offset is then the true applied micro-contact force. The LabVIEW program, specifically the virgin contact test, was designed for the user to input a step size in nanometers, a maximum applied force in micro-Newtons, and time interval for each step in milliseconds. For this research, the virgin contact test was applied for every cycle where a measurement was required. The user set the appropriate step size, time interval, and maximum applied force and then the system incremented the force sensor in the set step size until the force sensor limit was reached. At each increment, voltage, current, and force are recorded.

\section{2) Hot-Switch Testing}

The hot-switch test applies a given current while actuating the micro-contact open and closed for a given number of iterations. The user is required to input the maximum applied contact force, the number of cycles, the applied current, as well as the number of cycles between measurements. With the given information, the program applies the set level of current and actuates the micro-contact open and closed for the set number of cycles. On the cycle for which a measurement is to be made, the current, voltage, and applied force is recorded and placed in an excel file.

\section{3) Cold-Switch Testing}

Using the information from the virgin contact test, the user may opt to perform a cold-switch test instead of the hot-switch test. Cold-switching, as stated earlier, promotes the failure of the micro-contact through mechanical failure by applying current only when contact is already in 'make' and removing current before contact 'break'; thereby reducing the probability of electrical failure modes. While the inputs the user must provide are similar to the hot-switch test, the method of test is different in a significant way. For the coldswitch program, the micro-contact is first closed before current is applied. With the contact closed, the desired current level is applied to the micro-contact and the measurement of micro-contact current and voltage is then made. After the measurement of current and voltage, the applied current is then turned off and the micro-contact is opened. These steps are then repeated for the desired number of cycles.

\section{Micro-COnTACt Support Structure}

As mentioned previously, the fixed-fixed beam microcontact structure emulates Holm's crossed bar experiment on the micro-scale. This design allows a four-wire measurement in which current only flows through the micro-contact interface. Voltage is then measured across the micro-contact via gold traces connected to the anchor of the beam and micro-contact area.
Fixed-fixed beams all have a width of $450 \mu \mathrm{m}$, a length of $400 \mu \mathrm{m}$ and a beam thickness of $2.7 \mu \mathrm{m}$. They are designed with a gap of $3 \mu \mathrm{m}$ between the contact bump and contact pad. Sets of beams are fabricated with a contact bump with a radius of curvature ranges from $4 \mu \mathrm{m}, 6 \mu \mathrm{m}$, and $8 \mu \mathrm{m}$ at a depth of 1 $\mu \mathrm{m}$ from the beam. Sets of beams were also fabricated for each length and each contact bump radius which vary the number of contact bumps from one bump, two bumps, and three bumps. Fig. 2 shows a 3D model of the fixed-fixed beam micro-contact structure.

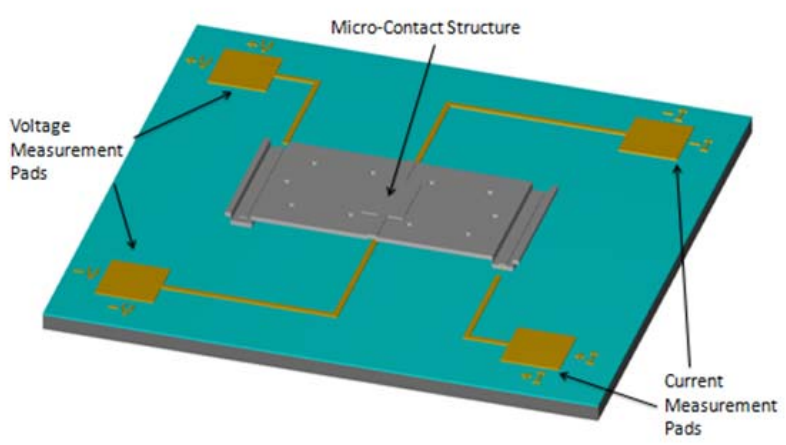

Fig. 2 3D Model Of Fixed-Fixed Beam Micro-Contact Support Structure

The micro-contact structure is constructed for gold-gold micro-contact and utilizes a structural layer to enhance the micro-contact structure's stiffness and to reduce the risk of stiction, but can be easily modified to investigate other contact materials. The advantage of the micro-contact structure's architecture is the ability to fabricate structures with different micro-contact materials to investigate the properties and physics of various contact materials and or structural layer materials to increase or decrease the beam stiffness to account for adhesion. The fixed-fixed beam design allows for thinner structural layers which can easily be surface micro machined where as a cantilever based design would require thicker films in order to have the same stiffness. In addition to fabricating entirely gold micro-contact support structures, other structures were fabricated that contained silver colloids $(15 \mathrm{~nm} \mathrm{Ag}$ spheres) embedded in the contact layer.

Silver colloids were added to the beam structure of the device by 'encasing' them in a thin sputtered layer of gold for the contact, applying the colloids, and electroplating gold over them. To remove any confusion, the resultant device is not an $\mathrm{Ag}-\mathrm{Au}$ or alloy type structure; it is an $\mathrm{Au}$ structure with encapsulated Ag colloids in the contact layer. For the purposes of this paper the resultant device will be referred to as the Ag$\mathrm{Au}$ beam. Fig. 3 shows an example diagram of an $\mathrm{Ag}-\mathrm{Au}$ device cross-section. The silver colloids can, in effect, change the paths of conduction through the contact since it is no longer uniform. As seen in the literature, creating a structure with two different metals does not always produce an alloy or composite which has better or even comparable performance to the individual constituents [5]. 


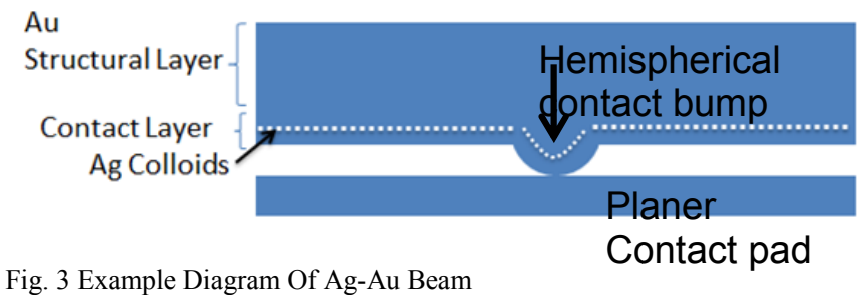

By itself, silver is a better conductor than gold, both thermally and electrically [4]. After deposition of the colloids, a thermal image was taken to demonstrate the thermal conductivity of Ag colloids. The addition of colloids may not only enhance the thermal conductivity of the micro-contact support structure, which would help maintain lower contact temperatures. Despite silver having a lower resistivity, a potential drawback to the design is the increased resistivity due to the Ag colloids changing the conducting paths of the electrons. As can be seen by Fig. 4, the more readily identifiable Ag Colloid groupings or 'clumps' appear as bright white, indicating better thermal conduction than the dark blue nitride coated silicon substrate and Au bottom metal layer.

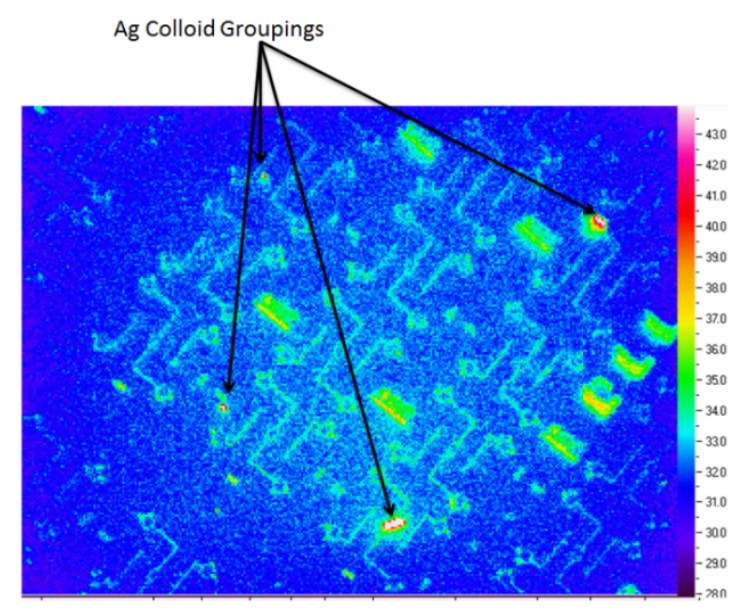

Fig. 4 Thermal Image Of Ag Colloids With Sample Heated To $60^{\circ} \mathrm{C}$

\section{Micro-CONTACT Physics}

Despite the surfaces of the contacts appearing similar, they are actually very different. When two surfaces meet, and because no surface is perfectly smooth, asperity peaks or "aspots", from each surface meet at the interface and form contact areas. Asperities have been described as "small cold welds providing the only conducting paths for the transfer of electrical current" [4]. Fig. 5 shows a graphical representation of apparent contact area, contacting a-spots, and the effective radius of the actual conducting area. The effective area is used for making simplified contact resistance calculations. Holm also investigated contact resistance changes due to plastic and elastic deformation of a-spots; which greatly affects the interface of the contact areas.

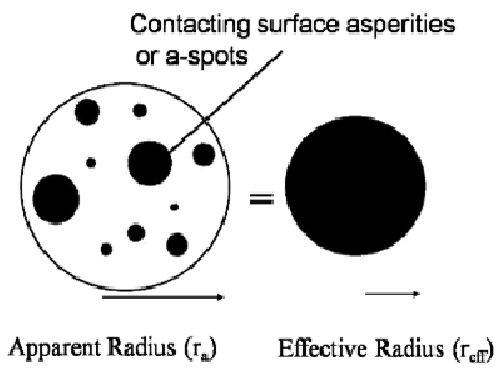

Fig. 5 A-spots As An Effective Radius [4]

As previously mentioned, the micro-contact resistance is the parameter being used to characterize the evolution of the performance of the micro-contact over its lifetime. From the literature, asperities on the surface of two materials are the first to make initial contact and allow current to flow through the micro-contact [3]. For simplified contact resistance calculations, the effective contact radius is used. The effective contact radius changes based on elastic, elastic-plastic, and plastic deformations caused by the applied force [3]. For micro-contacts, consideration of the electron's elastic mean free path $l_{e}$ compared to the effective radius $r_{e f f}$ is important for determining the appropriate analytical contact resistance equation. If the elastic mean free path is greater than the effective contact radius, $l_{e}>r_{e f f}$ then electrons are transported ballistically through the contact materials [7]. Alternatively, if $l_{e} \ll r_{e f f}$ then electrons are transported diffusively [7].

Elastic modeling is accurate for extremely low values of contact force where surface asperities retain their physical forms after the contact force is removed [8]. Elastic-plastic deformation occurs at the boundary between the permanent plastic deformation and the temporary elastic deformation [8]. Under plastic deformation, permanent surface change occurs by the displacement of atoms in asperity peaks whereas neighboring atoms are retained under elastic deformation [8].

\section{A. Elastic}

Asperity contact area under elastic deformation is given by [9]:

$$
A=\pi R \alpha
$$

Where $A$ is contact area, $R$ is asperity peak radius of curvature, and $\alpha$ is asperity vertical deformation. Hertz's model for effective contact area for elastic deformation as:

$$
r_{\text {eff }}=\sqrt[3]{\frac{3 F_{c E} R}{4 E^{\prime}}}
$$

where $E^{\prime}$ is the Hertzian modulus derived from:

$$
\frac{1}{E^{\prime}}=\frac{1-v_{1}^{2}}{E_{1}}+\frac{1-v_{2}^{2}}{E_{2}}
$$

with $E_{1}$ as the elastic modulus for contact one, $v_{1}$ is Poisson's ratio for contact one, $E_{2}$ as the elastic modulus for contact two, $v_{2}$ is Poisson's ratio for contact two [10,5]. Majumder et 
al. then related the contact area radius to contact force with [10]:

$$
F_{c E}=\frac{4}{3} E^{\prime} \alpha \sqrt{R \alpha} .
$$

The model for contact resistance for elastic deformation for diffusive transport is then [8]:

$$
R_{C D E}=\frac{\rho}{2} \sqrt[3]{\frac{4 E^{\prime}}{3 F_{c} R}}
$$

\section{B. Plastic}

To account for the asperity contact area and force under plastic deformation, the model from Abbot and Firestone that assumes sufficiently large contact pressure and no material creep is used [11]. Single asperity contact area and effective contact area are defined using (6) and (7) [5]:

$$
\begin{gathered}
A=2 \pi R \alpha \\
r_{e f f}=\sqrt{\frac{F_{c P}}{H \pi}}
\end{gathered}
$$

where $H$ is the Meyer hardness of the softer material [5], $A$ is contact area, $R$ is asperity peak radius of curvature, and $\alpha$ is asperity vertical deformation [5]. The effective contact area radius is then related to contact force by [3]:

$$
F_{c P}=H A \text {. }
$$

The model for contact resistance based on plastic deformation for diffusive transport is then [8]:

$$
R_{C D P}=\frac{\rho}{2} \sqrt{\frac{H \pi}{F_{c P}}}
$$

where $\rho$ is the resistivity of the conducting material, $H$ is the hardness of the material, $F_{c}$ is the contact force.

\section{Resistance Modeling}

For the $4 \mu \mathrm{m}, 6 \mu \mathrm{m}$, and $8 \mu \mathrm{m}$ contact radii, the microcontact resistance for the plastic and elastic deformation model is calculated using the material properties in Table 1.

Table 1 Material Properties For Au For Use In Resistance Modeling

\begin{tabular}{|lr|l|}
\hline \multicolumn{3}{|c|}{ Material properties for $\mathrm{Au}$} \\
\hline $\begin{array}{l}\text { Resistivity } \quad(\rho) \\
(\mathrm{Ohm}-\mu \mathrm{m})\end{array}$ & 0.022 \\
\hline $\begin{array}{l}\text { Hardness } \\
(\mathrm{Gpa})\end{array}$ & $(H)$ & 2.01 \\
\hline Poissons $\quad(v)$ & 0.42 \\
\hline $\begin{array}{l}\text { Young's modulus } \\
(\mathrm{GPa})\end{array}$ & 78 \\
\hline
\end{tabular}

\section{ANALYSIS AND RESULTS}

Each micro-contact support structure tested was subjected to the following tests as shown in Table 2. The tests were repeatable with minimal interruption caused by output errors from the current source. The current was kept constant at $0.03 \mathrm{~mA}$ and the voltage was measured to calculate the contact resistance. The devices were tested to examine the evolution of contact resistance over 10 million cycles. Measurements were made up to the designated number of cycles by the measurement interval. Between measurements, the microcontact was cycled at the actuation rate and force. This system has proven to be an effective test fixture for cycling micro-contacts at relatively fast cycle rates to examine the evolution of micro-contact resistance.

Table 2 Automated Micro-Contact Tests Performed by Micro-Contact Test Fixture

\begin{tabular}{|c|c|c|c|}
\hline $\begin{array}{c}\text { Number of } \\
\text { Cycles }\end{array}$ & $\begin{array}{c}\text { Measurement } \\
\text { Interval }\end{array}$ & $\begin{array}{c}\text { Actuation } \\
\text { Rate }(\mathrm{Hz})\end{array}$ & $\begin{array}{c}\text { Actuation } \\
\text { Force }(\mu \mathrm{N})\end{array}$ \\
\hline 100 & 10 th & 10 & 100 \\
\hline 1000 & 100 th & 100 & 100 \\
\hline 10000 & 1000 th & 1000 & 100 \\
\hline 100000 & 10000 th & 3000 & 100 \\
\hline 10000000 & 100000 th & 3000 & 100 \\
\hline
\end{tabular}

\section{A. Virgin Contact Test}

Fig. 6 shows a comparison of the measured micro-contact resistance for an $\mathrm{Au}$ only fixed-fixed micro-contact support structure, $2.7 \mu \mathrm{m}$ thick with a contact radius of $8 \mu \mathrm{m}$, and the predicted values of micro-contact resistance based on the equations in Section IV.

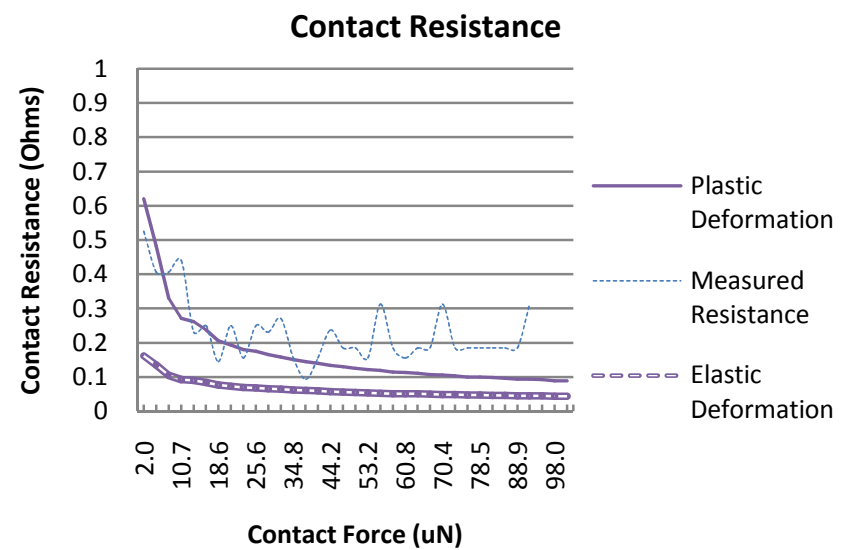

Fig. 6 Contact Resistance Of Au-Au Contacts

As seen in Fig. 6, the measured resistance value is in close agreement with the plastic deformation model. The initial contact resistance of less than $50 \mu \mathrm{N}$ is close to the plastic deformation resistance model and then begins to deviate after $50 \mu \mathrm{N}$. Variations between the modeled micro-contact resistance and measured results can be explained by the 
accuracy, precision, and performance of the test taking equipment.

Fig. 7 also shows a comparison of the measured microcontact resistance for an $\mathrm{Ag}$-Au fixed-fixed micro-contact support structure, $2.7 \mu \mathrm{m}$ thick with a contact radius of $8 \mu \mathrm{m}$, and the predicted values of micro-contact resistance based on based on the equations in Section IV. Similarly, the initial contact resistance of less than $25 \mu \mathrm{N}$ is close to the plastic deformation resistance model and then begins to deviate after $25 \mu \mathrm{N}$. The probable cause for this deviation is likely the resolution of the meters employed by the system.

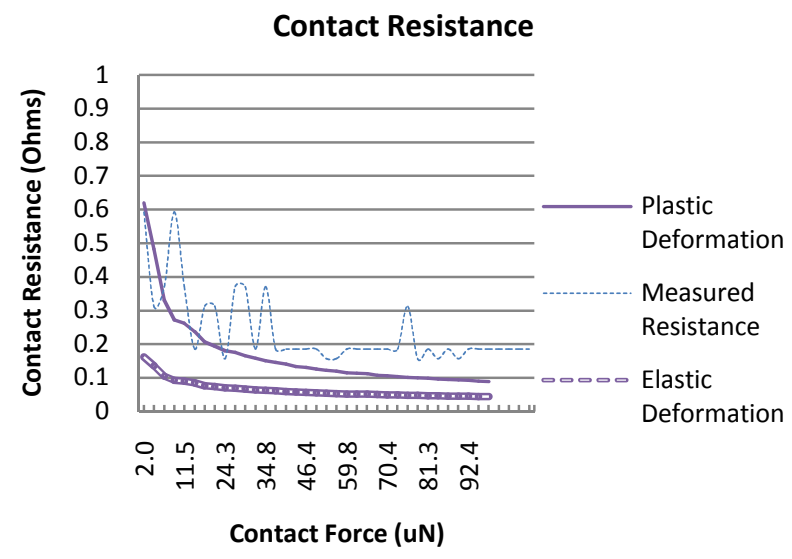

Fig. 7 Contact Resistance Of Ag-Au Contacts

\section{B. Cold-Switch Test}

For the cold-switch test (CST), the devices under test were first cycled up to 100 times at $10 \mathrm{~Hz}$ with measurements taken at every 10 cycles. Following the initial 100 cycles, the devices were actuated up to 1,000 cycles at $100 \mathrm{~Hz}$ with measurements performed every 100 cycles. Then 10,000 cycles at $1 \mathrm{kHz}$ with measurements every 1,000 cycles. In addition, the micro-contact structures were actuated to 100,000 cycles at $3 \mathrm{kHz}$ with measurements every 10,000 cycles. Lastly, the devices were actuated to 10 million cycles with measurements at every 100,000 cycles.

Fig. 8 shows the CST data collected for the fixed-fixed $\mathrm{Au}$ micro-contact support structure. The force required for contact make decreases from $220 \mu \mathrm{N}$ to $175 \mu \mathrm{N}$ after 1 million actuations. The cause of reduced required force for contact make is a change in the micro-contact support structure's shape. Using a Zygo white light interferometer, the tested micro-contact support structure was inspected for a visible change in structural architecture. As seen by Fig. 9 and Fig. 10, the force sensor indented the top of the Au beam of the micro-contact support structure.

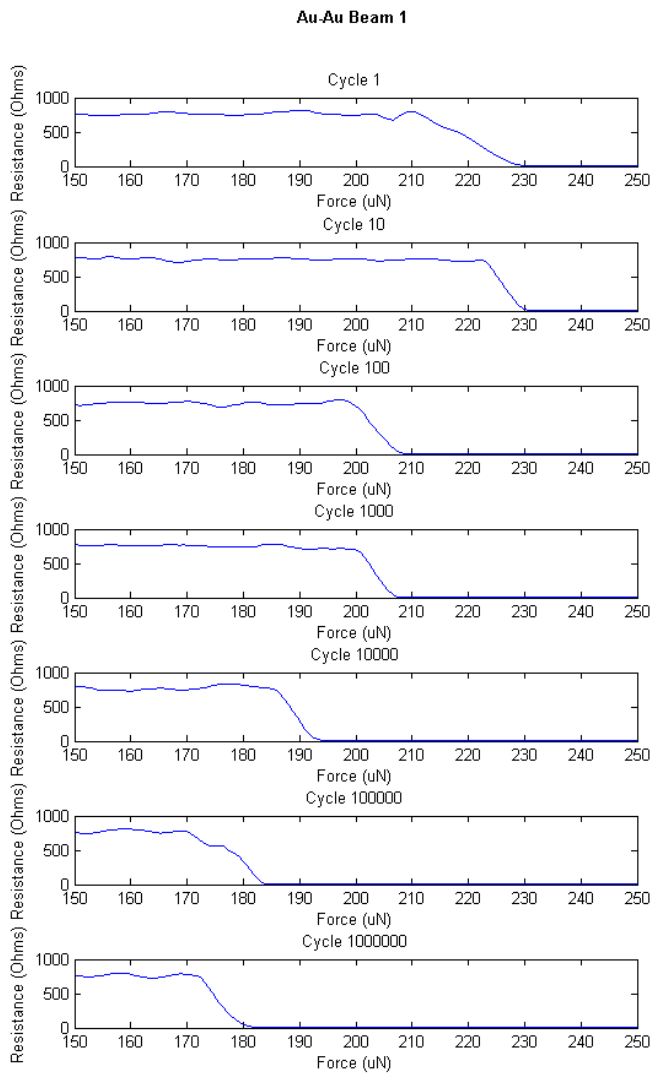

Fig. 8 Fixed-Fixed Au Micro-Contact Support Structure

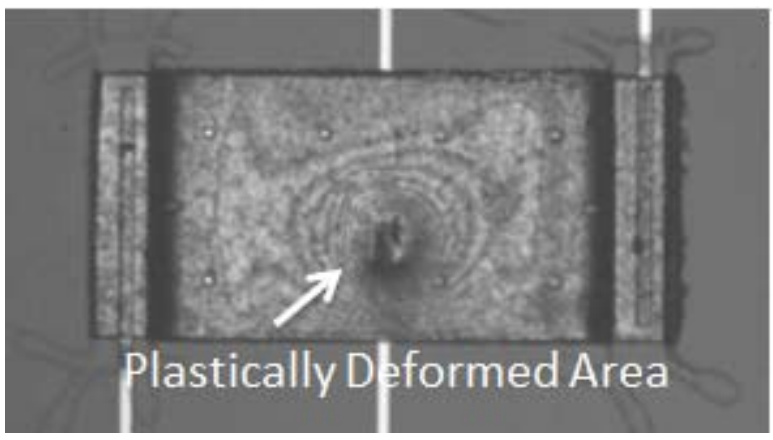

Fig. 9 Zygo Intensity Map showing Contour of Micro-Contact Support Structure 


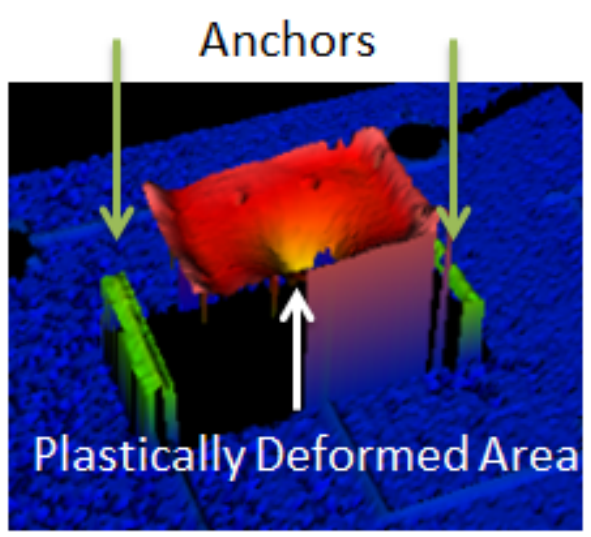

Fig. 10 Zygo 3D Image Of Fixed-Fixed Au Micro-Contact Support Structure

For plastic deformation of the support structure beam, the applied force must meet and surpass the yield stress of the material, which is the lowest value for stress to cause permanent deformation. According to Volinksky et al., the yield stress for $\mathrm{Au}$ films decreases with a rise in material temperature, which can increase by the flow of current through the micro-contact and supporting structure [12]. His team also reports that for a $2.7 \mu \mathrm{m}$ thick $\mathrm{Au}$ film, the yield stress can be as low as $360 \mathrm{MPa}$ (or $360 \mu \mathrm{N} / \mu \mathrm{m}^{2}$ ) at room temperature and become $160 \mathrm{MPa}\left(\right.$ or $160 \mu \mathrm{N} / \mu \mathrm{m}^{2}$ ) at $120^{\circ} \mathrm{C}$ [12]. Even though this testing was done at room temperature, localize hot spots during testing could have led to a lower yield stress. Considering that the max applied force to the top of the beam was $350 \mu \mathrm{N}$ in order to achieve near $100 \mu \mathrm{N}$ of contact force, it is likely that the yield stress threshold was passed causing the beam to be plastically deformed.

\section{Micro-Contact Resistance Evolution during CST}

Fig. 11 shows that the fixed-fixed $\mathrm{Au}$ micro-contact support structure experienced steadily increasing contact resistance for the first million actuations during cold switch testing. After contract resistance peaked at $17.87 \Omega$, there was a wear in period where contact resistance decreased to $3.88 \Omega$. This particular micro-contact was cycled nearly 10.2 million times, at which point the closed contact resistance was $14.43 \Omega$. Zygo imaging revealed that the micro-contact support structure beam had plastically deformed. The rise in resistance after 7 million cycles is caused by a change of the contact interface, possibly by changes through plastic deformation of the contact materials or development of a contaminate film.

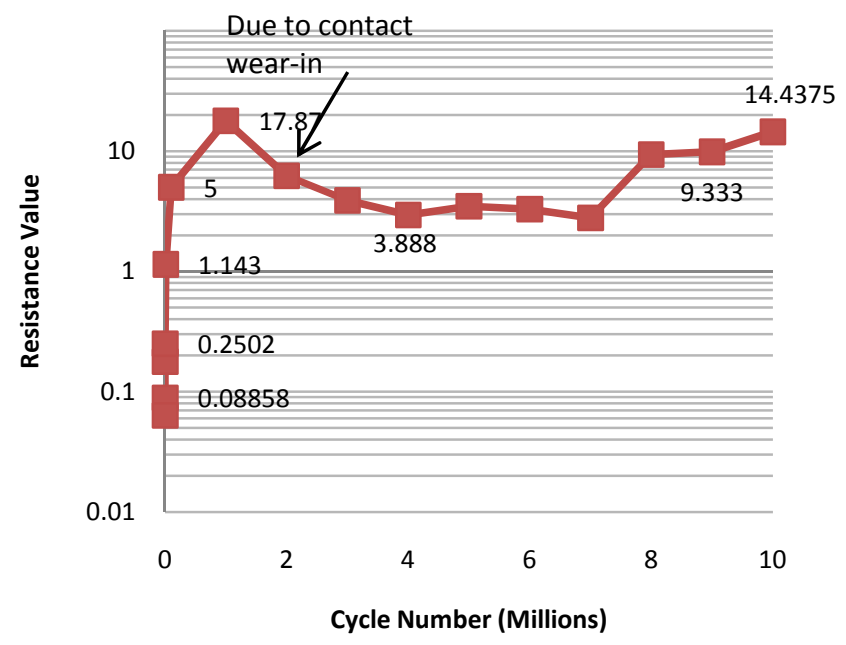

Fig. 11 Resistance Values During Cold Switch Testing For Fixed-Fixed Au Micro-Contact Support Structure

After cold-switching the functional fixed-fixed Au microcontact support structures for several million cycles a piece, the structures were 'peeled' back in order to reveal the lower contact area. The exposed lower contact areas were examined under the SEM for visual confirmation of changes to the contact area. Fig. 12 show the micro-contact areas after 4 million cycles. Examining Fig. 12, the micro-contact area shows evidence of material transfer and smooth bumps. The micro-contact area is not symmetrical due to changes in the micro-contact support structure over repeated actuations; deformation of the fixed-fixed beam changed the position of contact make. The asperities of the micro-contact likely experienced localized high temperatures from the current flow during the measurement. The raise in temperature at the localized spots caused the $\mathrm{Au}$ material to soften and in conjunction with mechanical pressure from the upper contact, smoothed the 'bumps' of the contact region.

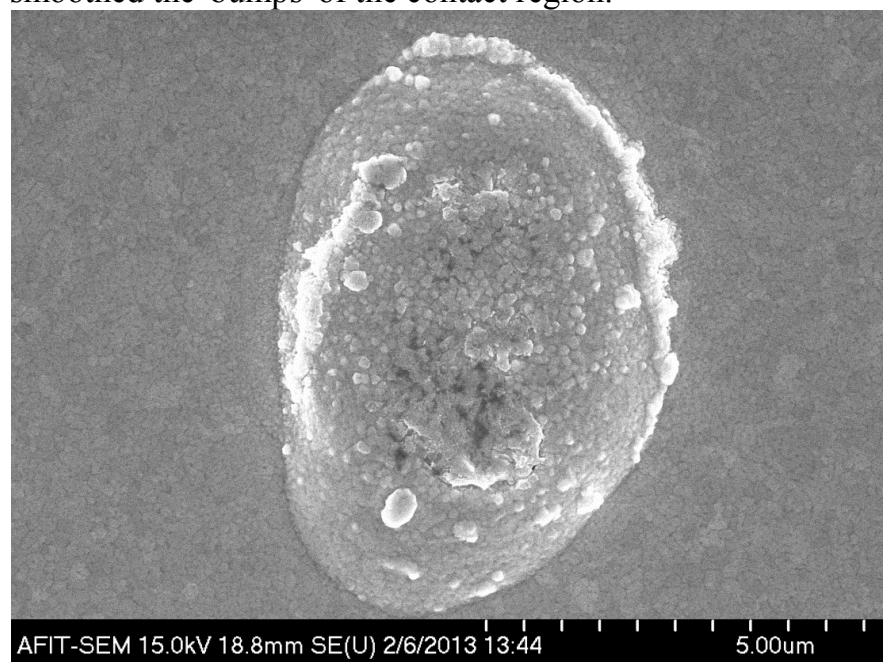

Fig. 12 Exposed Micro-Contact Area For Fixed-Fixed Au Micro-Support Structure 
The Ag-Au micro-contact support structure data shown in Fig. 13 shows that the micro-contact resistance varied from $0.238 \Omega$ to $4 \Omega$, improved from cycles 1000 to 10,000 , and then began to sharply rise after cycle 10,000 . The sharp rise in contact resistance can again be explained by the physical deformation of the contact support structure and micro-contact surface topology, which in turns can have a limiting effect on the area available for contact. In addition, the resistance over 2 million actuations is much higher than seen by the strictly $\mathrm{Au}$ device in Fig. 11. It is possible that the paths for current flow are constricted by the silver colloids in the contact layer. The colloids may be an obstruction to current flow because of the impedance mismatch between the encased colloids and surrounding gold film. Again, a characteristic drop in resistance near 5 million cycles occurs and then a drastic rise in resistance until failure (in this case the micro-contact remained closed at 8 million cycles). As discussed with the other micro-contact, there are many reasons for this resistance increase to occur. These reasons include plastic deformation of the contact interface, or development of a contaminate film.

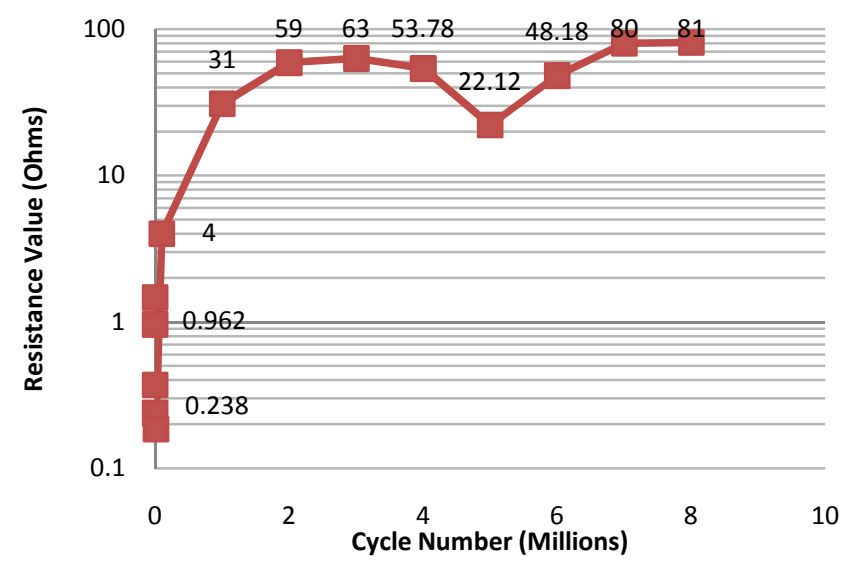

Fig. 13 Closed Resistance Values During Cold Switch Testing For FixedFixed Ag-Au Micro-Contact Support Structure

\section{CONCLUSIONS}

The system used in this research provides AFIT the unique capability to study the lifetime evolution of micro-contact performance using high cycle rates (up to $3 \mathrm{kHz}$ ), where previous cycle rates were reported at $800 \mathrm{~Hz}$ [1]. This paper reported the contact resistance evolution results of thin film, sputtered and evaporated gold, and Ag colloids encased micro-contacts dynamically tested up to $3 \mathrm{kHz}$.

\section{ACKNOWLEDGMENT}

The authors would like to thank Dr. L. Starman, Dr. Derrick Langley and Brent Danner for their support and assistance with theory, analysis, and fabrication. The authors would also like to extend gratitude to AFIT technicians, Mr. Rich Johnston and Mr. Tom Stephenson for their assistance and technical expertise.

\section{WORKS CITED}

[1] L. Chen, Z. J. Guo, N. Joshi, H. Eid, G. G. Adams and N. E. McGruer, "An improved SPM-based contact tester for the study of microcontacts," Journal of Micromechanics and Microengineering, vol. 22, p. 045017, 2012.

[2] Yang Z, Lichtenwalner D, Morris A, Menzel S, Nauenheim C, Gruverman A, Krim J, Kingon A, "A new test facility for efficient evaluation of MEMS contact," Journal of Micromechanics and Microengineering, vol. 17, pp. 1788-1795, 2007.

[3] Holm, R, Electric Contacts: Theory and Applications, Fourth Edition ed., Berlin: Springer, 1967.

[4] Braunovic M, Konchits V, Myshkin N, Electrical Contacts- Fundamentals, Applications, and Technology, New York: CRC Press, 2007.

[5] Coutu R, Reid J, Cortez R, Strawser R, Kladitis P, "Microswitches with Sputtered Au, AuPd, Au-on-AuPt, and AuPtCu Alloy Electrical Contacts," IEEE Transactions on Components and Packaging Technologies, vol. 29, no. 2, pp. 341-349, 2006.

[6] Timsit, R, "Constriction Resistance of Thin Film Contacts," IEEE Transactions on Components and Packaging Technologies, vol. 33, no. 3, pp. 636-642, 2010.

[7] Agrait N, Yeyati A, Ruitenbeek J, "Quantum Properties of atom-sized conductors," Physics Reports, vol. 377, pp. 81-279, 2003.

[8] Coutu R, McBride J, Starman L, "Improved MicroContact Resistance Model that considers Material Deformation, Electron Transport and Thin Film Characteristics," in Proc. 55th IEEE Holm Conference on Electrical Contacts, Vancouver, British Columbia, Canada, pp. 296-299, 2009.

[9] Chang W, "An elastic-plastic model for a rough surface with an ion-plated soft metallic coating," Journal of Wear, vol. 212, pp. 229-237, 1997.

[10] Majumder S, McGruer N, Adams G, Zavracky P, Morrison R, Krim J, "Study of contacts in an electrostatically actuated microswitch," Sensors Actuators, vol. 93, no. 1, pp. 19-26, 2001.

[11] Firestone F, Abbot E, "Specifying surface quantity - a method based on the accurate measurement and comparison," ASME Mech. Eng, vol. 55, p. 569, 1933.

[12] Volinsky A, Moody N, Gerberich W, "Nanoindention of $\mathrm{Au}$ and $\mathrm{Pt} / \mathrm{Cu}$ thin films at elevated temperatures," $J$. Mater. Res., vol. 19, no. 9, pp. 2650-2657, 2004. 